\title{
Research on EKF-Based Localization Method of Tracked Mobile Robot Junsuo Qu ${ }^{2, a}$,Qipeng Zhang ${ }^{1, b}$,Leichao Hou ${ }^{1, c}$, Ruijun Zhang ${ }^{1, d}$,kaiming Ting ${ }^{3, e}$

\author{
${ }^{1}$ Innovation Lab, Xi'an University of Posts and Telecommunications, Xi'an 710061, P. R. China; \\ 2Institute of IOT \& IT-based Informatization, Xi'an 710061, P. R. China; \\ University Australia.
} \\ ${ }^{3}$ Centre of Multimedia Computing, Communications, and Artificial Intelligence Research Federation
}

aqujunsuo@qq.com,b374387267@qq.com,c1457633811@qq.com, ${ }^{\text {d } 472780551 @ q q . c o m, ~}$

ekaiming.ting@federation.edu.au

Keywords: Mobile robot, position ,heading angle ,Extended Kalman Filtering , Odometry.

\begin{abstract}
To estimate the position and heading angle of mobile robot precisely, an measurement variable estimation model was proposed to adapt any angle. Fusing the predictive value of odometry and measurement data of multiple sensors by the Extended Kalman Filtering (EKF) for reducing the accumulative error by using only traditional odometry. The proposed models is verified by Matlab simulation and experimental results.
\end{abstract}

\section{Introduction}

Autonomous mobile robot's position determination has been the subject of intelligent field, if mobile robot know its position and heading angle timely, it is a prerequisite in executing given tasks [1].The recent progress in sensor technologies and increase in microcontroller unit's computational power brings great experimental platform for research on localization of autonomous robots[2].The usual method for position and heading angle estimation of a mobile robot is using only odometry. This method using the data obtained from the mobile sensor like encoder to estimate the change of the position of mobile robot with time. However, in the process of localization of mobile robot, it has a inevitable problem of accumulative error when the mobile robot moves long distances[3-4].To improve the accuracy of odometry using in localization estimation of mobile robot, many research have been undergone in the filed of eliminate the systematic error, robot design constraints and environment influences. A common approach consists of two parts. First part consists of a better error or odometry model and second part consists of using additional sensors.

The Kalman Filtering considers the influence of process noise and measurement noise, can obtain the optimal estimation of the state variables of the linear system[6],is very often used to solve the localization problem in mobile robot. Since the Extended Kalman Filtering (EKF) algorithm is a well proven and commonly used in research of mobile robot's position determination [7-9],has a strong ability to restrain interference and suit for the optimal estimation of nonlinear systems.

This paper is organized as follows. In Section 1 the kinematics model of the mobile robot are described. In Section 2 the measurement mode of the mobile robot's multiple sensor system are described. In Section 3 the prediction step and the correction step of the EKF are described. In Section 4 are the results of localizing the mobile robot in an laboratory. The paper is concluded in Section 5.

\section{Kinematics Model}

The method of estimate mobile robot's localization introduced in this paper need to obtain the two independently speed-controlled wheel's angular velocities based on encoders of the multiple sensor system. The kinematics model is established by using odometry, equation(1). The mobile robot is assumed to be traveling on a flat surface and ignore the question of wheel slippage. To describe mobile robot's kinematics model in this paper, establish the global coordinates $X_{G} O_{G} Y_{G}$ and the 
robot coordinates $X_{L} O_{L} Y_{L}$ (Fig. 1), the mobile robot's position and heading angle are defined by $X(k)$ at time step $\mathrm{k}$ according to the global coordinates.

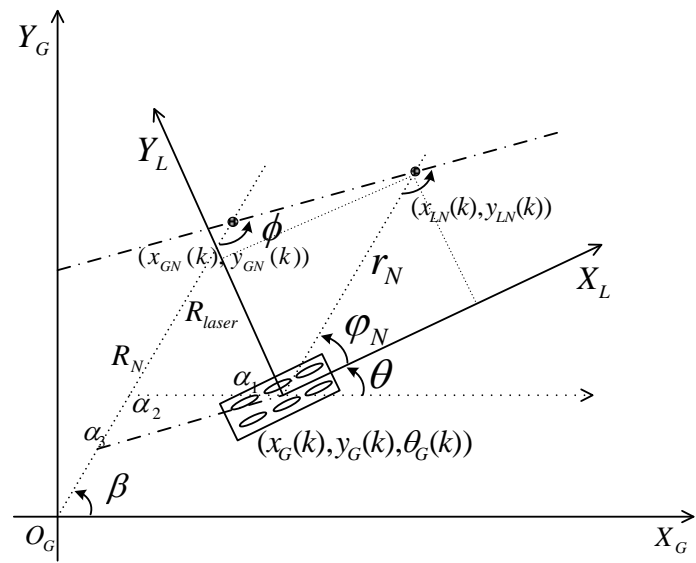

Fig. 1 Mobile robot's localization according to the global coordinates $X(k)=f[X(k-1), K-1]+\Gamma_{k-1} W(k-1):$

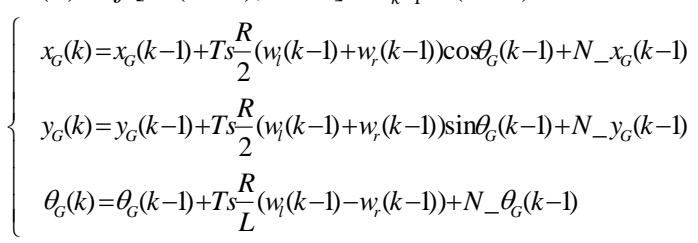

The mobile robot's location result is defined by $X(k)=\left[x_{G}(k), y_{G}(k), \theta_{G}(k)\right]^{T}$ at time step $\mathrm{k}$ according to the global coordinate frame, where $\left.\left[x_{G}(k), y_{G}(k)\right)\right]^{T}$ specifies the robot's position in the rectangular coordinate, $\theta_{G}(k)$ specifies the robot's heading angle. $\mathrm{R}$ is the radius of robot's wheel, $\mathrm{L}$ is the length of mobile robot. $W(k-1)=\left[N_{-} x_{G}(k-1), N_{-} y_{G}(k-1), N_{-} \theta_{G}(k-1)\right]^{T}$ denotes uncorrelated zero-mean process noise, $\Gamma_{k-1}$ denotes the transformation matrix of process noise. $w_{l}(k)$ and $w_{r}(k)$ denotes the angular velocities of the mobile robot's left and right wheel, $T_{s}$ is the sampling period.

\section{Measurement Model}

The VL53L0X is the smallest time-of-flight (ToF) sensor on the market today, absolute distance provided (in $\mathrm{mm}$ ) up to 2 meters in less than $30 \mathrm{~ms}$. The obstacle in global environment is assumed to a set of line segment, described with equation: $y=k_{n} x+b, k_{n}$ is the slope of the $n$th line segment. In laser-range-finder(LRF)-based measurement model, $\left(r_{N}, \varphi_{N}\right)^{T}$ denotes the Nth measurement value based on the LRF scan, $r_{N}$ is the distance between the mobile robot and the obstacle, $\varphi_{N}$ is the included angle between the servo motor and the robot coordinate's $\mathrm{x}$-axis.

The included angle between the origin of a global coordinate and the line segment is $\phi$, the measurements taken from VL53L0X is represented by $R_{N}=x_{G N}(k) \cos \beta+y_{G N}(k) \sin \beta$.In [3]the $\phi=90^{\circ}$ have been discussed in this paper, we discuss the $\phi$ in $\left[0^{\circ}, 180^{\circ}\right]$. We can approximate the measurement vector $h\left[\hat{X}_{k / k-1}\right]$ using global vector $\left[x_{G N}(k), y_{G N}(k), \beta\right]^{T}$ as

$$
\begin{aligned}
& l_{3}=x_{G}(k)-y_{G}(k) \tan (\beta) \\
& l_{1}=\sin (\arctan k)\left(l_{3} / \sin (\beta-\arctan k)\right) \\
& h\left[\hat{X}_{k / k-1}\right]=\left[\begin{array}{l}
R_{\text {laser }} \\
\varphi_{\text {laser }}
\end{array}\right]=\left[\begin{array}{l}
\left|R_{N}-\left(y_{G}(k) / \sin (\beta)-l_{1}\right)\right| \\
\beta-\theta_{\text {heading }}
\end{array}\right]
\end{aligned}
$$




\section{Extended Kalman Filtering}

To estimate the state of a nonlinear stochastic system from noisy measurements, we can linearize nonlinear equation of kinematics model and measurement model of mobile robot with the Taylor series expansion. The EKF is a well proven and widely used state estimation algorithm, figure 2 shows the block diagram of the EKF-Based localization method of mobile robot in this paper. To fuse the information of Odometry predictions and measurement from sensors of multiple sensors by utilizing the EKF, obtain optimal estimation of position and heading angle of mobile robot by the algorithm of recursive. The EKF consists of a prediction and a correction step[10] and are defined as equation (3) and (4).

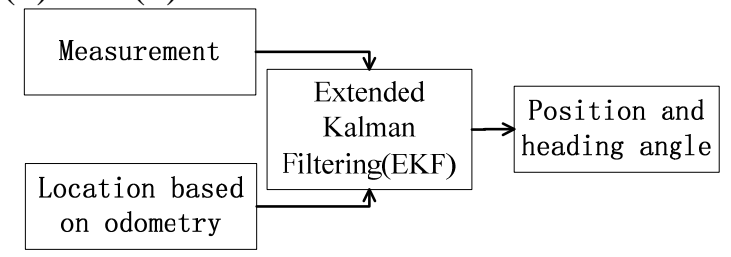

Fig. 2 the EKF-Based Localization Method of mobile robot

\subsection{Prediction Step of the EKF}

$$
\begin{aligned}
& \left\{\begin{array}{c}
\hat{X}_{k / k-1}=f[X(k-1), k-1]+\Gamma_{k-1} W(k-1) \\
\hat{P}_{k / k-1}=F_{k} P_{k-1} F_{k}^{T}+Q_{k-1}
\end{array}\right. \\
& F_{k}=\left.\frac{\partial f_{i}}{\partial X_{j}(k)}\right|_{\hat{X}_{k-1}}=\left[\begin{array}{l}
\frac{\partial x_{G}(k)}{\partial x_{G}} \frac{\partial x_{G}(k)}{\partial y_{G}} \frac{\partial x_{G}(k)}{\partial \theta_{G}} \\
\frac{\partial y_{G}(k)}{\partial x_{G}} \frac{\partial y_{G}(k)}{\partial y_{G}} \frac{\partial y_{G}(k)}{\partial \theta_{G}} \\
\frac{\partial \theta_{G}(k)}{\partial x_{G}} \frac{\partial \theta_{G}(k)}{\partial y_{G}} \frac{\partial \theta_{G}(k)}{\partial \theta_{G}}
\end{array}\right]
\end{aligned}
$$

$X(k-1)=\left[x_{G}(k-1), y_{G}(k-1), \theta_{G}(k-1)\right]^{T}$ above represents the optimal estimation at time step $\mathrm{k}$ after getting all the measurements from sensor, whereas $P_{k / k-1}$ is the covariance matrix of the estimation error. There $\hat{X}_{k / k-1}$ represents the estimation of $X_{k}$ before getting the measurement from sensor, $\hat{P}_{k / k-1}$ denotes the covariance matrix of the state-prediction error, $\Gamma_{k-1}$ is the transformation matrix of process noise.

In the kinematics model of mobile robot, $W(k)=\left[N_{-} x_{G}(k), N_{-} y_{G}(k), N_{-} \theta_{G}(k)\right]^{T}$ is the process noise with zero mean and represents the accumulative error from the kinematics model, the measurements error from multiple sensor system and robot's design constraints in odometry. $\sigma$ is the constant obtained during experiment when get the optimal estimation of location, $Q_{k}$ denotes covariance matrix of process noise $W_{k}$,

$$
Q_{k}=\left[\begin{array}{cccc}
\sigma N_{-} x_{G}{ }^{2}(k) & 0 & 0 \\
0 & \sigma N_{-}{ }_{G}{ }^{2}(k) & 0 \\
0 & 0 & \sigma N_{-} \theta_{G}{ }^{2}(k)
\end{array}\right]
$$

\subsection{Correction Step of the EKF}

$$
\begin{gathered}
\left\{\begin{array}{c}
K_{k}=P_{k / k-1} H_{k}^{T}\left(H_{k} P_{k / k-1} H_{k}^{T}+R_{k}\right) \\
P_{k}=\left(I-K_{k} H_{k}\right) P_{k / k-1} \\
X_{k}=\hat{X}_{k / k-1}+K_{k}\left(Z_{k}-h\left[\hat{X}_{k / k-1}\right]\right)
\end{array}\right. \\
H_{k}=\left.\frac{\partial h_{i}}{\partial X_{j}(k)}\right|_{\hat{X}_{k / k-1}}=\left[\begin{array}{l}
\frac{\partial r_{\text {laser }}}{\partial x_{G}} \frac{\partial r_{\text {laser }}}{\partial y_{G}} \frac{\partial r_{\text {laser }}}{\partial \theta_{G}} \\
\frac{\partial \varphi_{\text {laser }}}{\partial x_{G}} \frac{\partial \varphi_{\text {laser }}}{\partial y_{G}} \frac{\partial \varphi_{\text {laser }}}{\partial \theta_{G}}
\end{array}\right]
\end{gathered}
$$


The mobile robot's position and heading angle predicted by kinematics model is corrected by measurement, utilizing VL53L0X to obtain the distance between sensor equipped in robot and obstacles around $\left[0^{\circ}, 180^{\circ}\right] . \hat{X}_{k / k-1}$ and $\hat{P}_{k / k-1}$ are obtained in the prediction step of the EKF are corrected by the measurement $Z_{k}$, linearize nonlinear equation(2) and use EKF, $H_{k}$ is the Jacobian matrix of partial derivatives of with respect to $\left[x_{G}(k), y_{G}(k), \theta\right]^{T}$ at time step k.

Kalman gain $K_{k}$ serves to correctly update $\hat{X}_{k / k-1}$ which represents the estimation of $X_{k}$ and $\hat{P}_{k / k-1}$ which is the covariance matrix of the estimation error. $X_{k}$ above represents a posterior estimation of the state by updating $\hat{X}_{k / k-1}$ with the measurement $Z_{k}$ at time step $\mathrm{k}, P_{k}$ above denotes the error covariance updated with the kalman gain $K_{k}$ at time step k.

In the measurement model of mobile robot, $V(k)=\left[N_{-} r_{\text {laser }}(k), N_{-} \varphi_{\text {laser }}(k)\right]^{T}$ is the measurement noise with zero mean. $\lambda$ is the constant obtained during experiment when get the optimal estimation of location, $R_{k}$ denotes covariance matrix of process noise $V(k)$,

$$
R_{k}=\left[\begin{array}{lr}
\lambda N_{-} r_{\text {laser }}{ }^{2}(k) & 0 \\
0 & \lambda N_{-} \varphi_{\text {laser }}{ }^{2}(k)
\end{array}\right]
$$

\section{Experimental Verification}

\subsection{Experiment Platform}

The experiment platform for researching localization method of mobile robot is shown in figure 3.The LRF sensor VL53L0X of multiple sensors is equipped on the platform of servo motor whose rotation angle from $0^{\circ}$ to $180^{\circ}$.The LRF is used to scan the environment around mobile robot and obtain the distance between obstacle and mobile robot. The encoder is used to measure two independently speed-controlled wheel's angular velocities. The Bluetooth module is used to transfer date between multiple sensors and mobile robot.

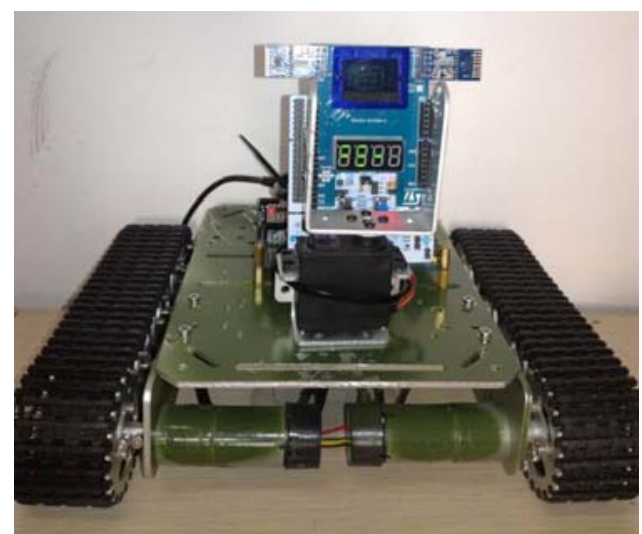

\subsection{Experimental Results}

Fig. 3 The Mobile Robot for Researching Localization Method

The experiment environment of research on EKF-Based localization method of tracked mobile robot according to the two-dimensional global coordinates is shown in figure 4, and rectangles in figure 4 represent the obstacle in the experiment environment. The black arrow denote moving track of the mobile robot. The mobile robot can obtain the obstacle information by using LRF sensor VL53L0X on the servo motor, and finish path planning by using Microcontroller Unit(MCU).

Noise variable in the kinematics model and measurement model are assumed to be uncorrelated white random noises. In order to obtain detailed position and heading angle of mobile robot, the sampling time in the experiment is $T_{s}=1 \mathrm{~s}$, using EKF to fuse the estimation of Odometry and measurement of multiple sensors with 300 points of location each time. The comparison between 
Odometry, actual value and estimation of EKF by using Matlab is shown in figure 5, figure 6 shows the error comparison between Odometry and EKF.

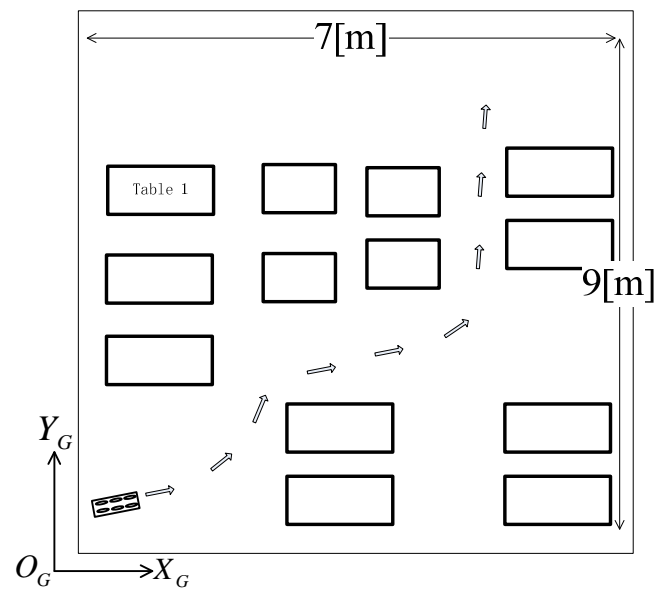

Fig. 4 The experiment environment for Researching Localization Method

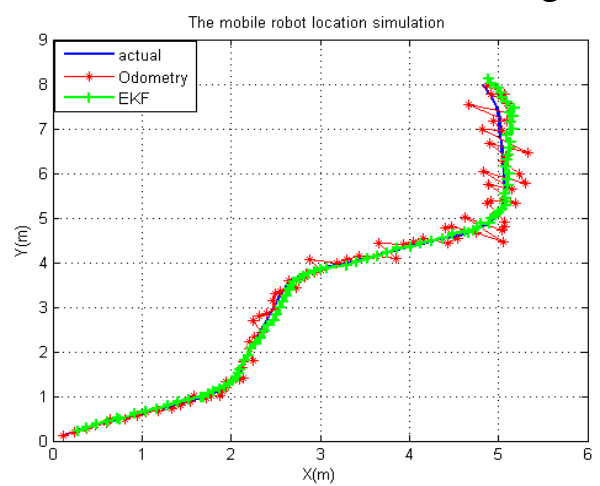

Fig. 5 Location comparison between actual, Odometry and EKF

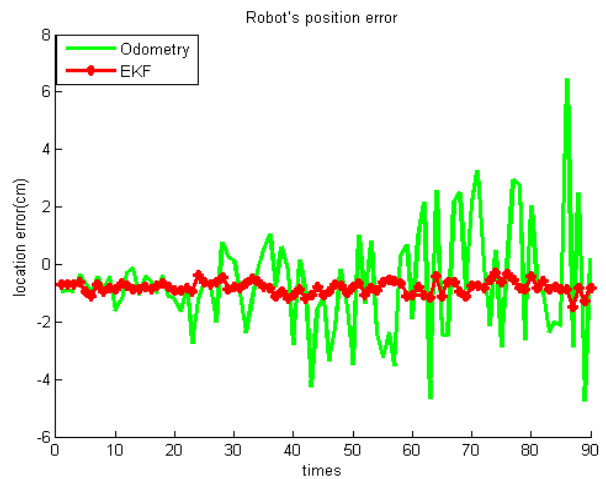

Fig. 6 Error comparison between Odometry and EKF

By using Matlab to obtain the experimental results comparison between different ways in this experiment mentioned above, we find that the traditional odometry used to obtain the position and heading angle of mobile robot have accumulative error at the same time, the value will gradually lose the reference value with the increase of the number of experiment, using EKF to fuse results of Odometry and measurement of multiple sensors can obtain the optimal estimation with the error less than $1.01 \mathrm{~cm}$,location accuracy is significantly improved compared with Odometry.

\section{Conclusion}

This paper specifically report the kinematics model and measurement model in the research of EKF-Based localization method. Then the newly implemented calculation model of measurement variables is proposed and described concretely, which is suitable for from $0^{\circ}$ to $180^{\circ}$. We can see from the experiment results that the the position and heading angle of mobile robot obtained by 
applying EKF can effectively reduce the accumulative error by traditional odometry. The experimental results show the practicability of calculation model and verify the location method of mobile robot.

\section{Acknowledgments}

This work is supported by Innovation Project of the Department of Science and Technology Shaanxi Province (2016ZCKJ3-090), Graduate Innovation Fund of Xi'an University of Posts \& Telecommunications (CXL2016-01).

\section{References}

[1]. Vladimir Kubelka, Michal Reinstein, Complementary Filtering Approach to Orientation Estimation using Inertial Sensors Only[C], IEEE International Conference on Robotics and Automation, May 14-18, 2012:599 - 605.

[2]. Simanek J, Reinstein M, Kubelka V. Evaluation of the EKF-Based Estimation Architectures for Data Fusion in Mobile Robots [J]. IEEE/ASME Transactions on Mechatronics, 2015, 20(2):985-990.

[3]. D Alfonso L, Lucia W, Muraca P, et al. Mobile robot localization via EKF and UKF: A comparison based on real data[J]. Robotics \& Autonomous Systems, 2015, 74:122-127.

[4]. Teslić L, Š krjanc I, Klančar G. EKF-Based Localization of a Wheeled Mobile Robot in Structured Environments [J]. Journal of Intelligent \& Robotic Systems, 2011, 62(2):187-203.

[5]. Ivanjko E, Petrovic I. Extended Kalman filter based mobile robot pose tracking using occupancy grid maps[C]// Electrotechnical Conference, 2004. Melecon 2004. Proceedings of the, IEEE Mediterranean. 2004:311-314 Vol.1.

[6]. Yongyuan Qin, Hongyue Zhang,Shuhua Wang. Calman. Principles of filtering and integrated navigation. (Third Edition) [M]., Northwestern Polytechnical University press.

[7]. Teslić L, Š krjanc I, Klančar G. Using a LRF sensor in the Kalman-filtering-based localization of a mobile robot [J]. Isa Transactions, 2010, 49(1):145-53.

[8]. Xiaoliang $\mathrm{Xu}$, HaoZhang, Xian Feng Tang. The integration of the micro sensor systems EKF noise correlation algorithm [J]. Journal of Tsinghua University (NATURAL SCIENCE EDITION), 2012 (9): 1199-1204.

[9]. Ma J, Susca S, Bajracharya M, et al. Robust multi-sensor, day/night 6-DOF pose estimation for a dynamic legged vehicle in GPS-denied environments[C]// IEEE International Conference on Robotics and Automation. IEEE, 2012:619-626.

[10]. Iv F P V, Lee D J. Design of an EKF-CI based sensor fusion for robust heading estimation of marine vehicle[J]. International Journal of Precision Engineering and Manufacturing, 2015, 16(2):403-407. 